\title{
In Vivo Evaluation of the Presence of Bone Marrow in Cortical Porosity in Postmenopausal Osteopenic Women
}

\author{
Janet Goldenstein, ${ }^{1,2}$ Galateia Kazakia, ${ }^{1}$ and Sharmila Majumdar ${ }^{1}$ \\ ${ }^{1}$ Musculoskeletal and Quantitative Imaging Research Group, Department of Radiology and Biomedical Imaging, University \\ of California, San Francisco, CA, USA; and ${ }^{2}$ UCSF-UCB Joint Graduate Group in Bioengineering, Berkeley, CA, USA
}

(Received 13 July 2009; accepted 17 November 2009; published online 2 December 2009)

\begin{abstract}
This is the first observational study examining cortical porosity in vivo in postmenopausal osteopenic women and to incorporate data from two different imaging modalities to further examine the nature of cortical porosity. The goal of this study was to combine high-resolution peripheral computed tomography (HR-pQCT) images, which contain high spatial resolution information of the cortical structure, and magnetic resonance (MR) images, which allow the visualization of soft tissues such as bone marrow, to observe the amount of cortical porosity that contains bone marrow in postmenopausal osteopenic women. The radius of 49 and the tibia of 51 postmenopausal osteopenic women (age $56 \pm 3.7$ ) were scanned using both HR-pQCT and MR imaging. A normalized mutual information registration algorithm was used to obtain a threedimensional rigid transform which aligned the MR image to the HR-pQCT image. The aligned images allowed for the visualization of bone marrow in cortical pores. From the HR-pQCT image, the percent cortical porosity, the number of cortical pores, and the size of each cortical pore was determined. By overlaying the aligned MR and HR-pQCT images, the percent of cortical pores containing marrow, the number of cortical pores containing marrow, and the size of each cortical pore containing marrow were measured. While the amount of cortical porosity did not vary greatly between subjects, the type of cortical pore, containing marrow vs. not containing marrow, varied highly between subjects. The results suggest that cortical pore spaces contain components of varying composition, and that there may be more than one mechanism for the development of cortical porosity.
\end{abstract}

Keywords-Cortical porosity, MRI, High-resolution pQCT, Image registration.

Address correspondence to Janet Goldenstein, Musculoskeletal and Quantitative Imaging Research Group, Department of Radiology and Biomedical Imaging, University of California, San Francisco, CA, USA. Electronic mail: janethb@berkeley.edu, galateia.kazakia@ radiology.ucsf.edu, sharmila.majumdar@radiology.ucsf.edu

This study uses data collected as part of a clinical trial study entitled "Alendronate Prevents Microarchitectural Deterioration of Trabecular Bone in Early Postmenopausal Women." The unique trial number is NCT00504166, and the trial registration date was July 17, 2007.

\section{INTRODUCTION}

Cortical bone has a complex structure that plays an important role in bone strength. ${ }^{2}$ Structure and mechanical properties of cortical bone have been reported to change due to age, ${ }^{13,27} \mathrm{sex},{ }^{21}$ and osteoporotic status. ${ }^{1}$ Recent studies have focused on porosity of the cortical bone. , 7,14,15 $^{\text {Macroporosity }}$ has been defined as porosity with a diameter greater than $0.385 \mathrm{~mm}$ and is reported to increase with age. ${ }^{7}$ Cortical porosity has a significant impact on mechanical properties of cortical bone. ${ }^{18,40,51,63}$ Yeni et al. reported that femoral and tibial cortical bone toughness decreases with increasing cortical porosity. ${ }^{63}$ Additionally Young's modulus, a measure of stiffness, and the ultimate stress of cortical bone have both been shown to decrease with increasing porosity. ${ }^{18,40,51}$ The exact mechanism behind the formation of cortical macroporosity is unknown. The existence of large cortical pores has been attributed to resorption spaces, merging of haversian canals, reduction in the rate of closure of haverian canals, and a clustering of osteons. ${ }^{4,14,28,39,47}$ The fluid contained within a cortical pore may be an indication of the cause of cortical pore formation. If the cortical pore is filled with fatty bone marrow then the surrounding cortical bone may be becoming more trabecular-like in structure and if the cortical pore is filled with blood vessels then the cortical pore is more likely to have been formed by a merging of haverian canals. Characterizing the fluid contained in cortical pores will enhance our understanding of the formation of cortical porosity and ultimately contribute to the ability to estimate bone strength and predict fracture risk.

Hydraulic strengthening (HS) of bone refers to the theory that pressure due to bone fluids found in the cavities of bone might hydraulically strengthen bone by reducing bone stresses during dynamic loading. If bone cavity fluid pressures are substantial, they may increase the load-bearing capacity of bone. HS of bone 
is controversial because direct experimental verification is currently untenable because fluid pore spaces are extremely small and encased in hard tissue. ${ }^{35} \mathrm{Sev}$ eral studies have estimated the fluid flow contribution or HS effect on trabecular bone mechanical properties ${ }^{29,30,45}$ and cortical bone apparent modulus. ${ }^{35}$ However, the precise nature (viscosity and compressibility) of bone fluid present in cortical porosity is unknown ${ }^{16}$ and accuracy of HS models depend greatly on defining appropriate bone pore fluid properties. ${ }^{35,36}$ The nature of the bone fluid may affect the bone's ability to withstand dynamic loading, therefore a more precise characterization of cortical bone fluid may help improve the accuracy of HS models.

The improvement in cortical thickness and cortical porosity due to antiresorptive therapies highlights the importance of cortical bone in overall bone quality maintenance. ${ }^{22,50}$ Roschger et al. found that cortical porosity of the iliac crest decreased from $7.5 \%$ in a placebo group to about $4 \%$ after alendronate treatment in postmenopausal osteoporotic women. ${ }^{50}$ Recently, Borah et al. reported a $18-25 \%$ reduction in cortical porosity of the iliac crest for postmenopausal osteoporotic women treated with risedronate. ${ }^{10}$ While there is a decrease in cortical porosity with antiresorptive therapies, it may be possible that the constituents of cortical pores further impact remodeling and therapeutic response. Therefore, characterizing cortical pore fluid may help to understand why cortical porosity has an enhanced response to antiresorptive therapy.

High-resolution peripheral computed tomography (HR-pQCT) is an emerging imaging technique which achieves an isotropic nominal resolution of $82 \mu \mathrm{m}$, allowing for visualization of cortical macroporosity in vivo at peripheral sites such as the tibia and radius. ${ }^{12,31}$ Magnetic resonance (MR) imaging is an imaging technique that allows visualization of soft tissues such as bone marrow. It is our hypothesis that by combining these two imaging techniques, visualization of bone marrow within cortical porosity is possible. Our goal is to observe the amount of cortical porosity that contains bone marrow in postmenopausal osteopenic women and determine if there is a relationship between pore size and the existence of bone marrow.

\section{METHODS AND MATERIALS}

\section{Subjects}

The subjects in this study were 52 postmenopausal women (mean age \pm standard deviation $=56 \pm 3.7$ ) who were recruited for a double-blind study investigating the longitudinal effects of alendronate vs. placebo on cortical and trabecular bone microarchitecture. Only women between the ages of 45 and 65 years that had been postmenopausal for at least 1 year but not more than 6 years were included. They were required to be defined as osteopenic by the WHO criteria $^{62}$ and had no history of disease or treatment known to affect bone metabolism. The study protocol was approved by the UCSF Committee on Human Research and all women provided written informed consent.

Images in this study were taken from the baseline time-point prior to alendronate treatment. Imaging using both HR-pQCT and MR was attempted on all 52 subjects. However, three of the subjects could not tolerate the prone position required for the MR radius scan and one tibia MR image was excluded from the study due to motion artifact and poor image quality. Therefore, this study analyzed radius images of 49 subjects and the tibia images of 51 subjects. While previous studies examining cortical porosity have focused on the midshaft, ${ }^{7,14}$ images in this study were acquired in a region allowing for both trabecular and cortical bone analysis while minimizing scan time and radiation dose.

\section{MRI}

MR images were acquired on a 3T (GE Signa) MR scanner. Images of the distal radius were acquired with the subject in a prone position using a transmit receive quadrature wrist coil (Mayo Foundation for Medical Education and Research) and images of the distal tibia were acquired with the subject in a supine position using a four-channel dual paddle coil (Nova Medical). Images were obtained with a balanced steady state free precession (bSSFP) pulse sequence. ${ }^{32}$ Pulse sequence parameters were $512 \times 384$ matrix, $60^{\circ}$ flip angle, $122 \mathrm{~Hz} /$ pixel BW, $8 \mathrm{~cm}$ FOV. The tibia images had a 16.8-17.8/6.5 $\mathrm{ms} \mathrm{TR} / \mathrm{TE}$ and scan time of $15 \mathrm{~min}$ and the radius images had a 14.4-15.21/3.3-3.9 ms TR/TE and scan time of $10 \mathrm{~min}$. The image acquisition resulted in an image resolution of $156 \mu \mathrm{m} \times 156 \mu \mathrm{m} \times$ $500 \mu \mathrm{m}$. Images were originally acquired with optimal scanning parameters for trabecular bone quantification. However, the bSSFP sequence also has superior signal-to-noise ratio and maximizes bone marrow signal. $^{3,32}$

\section{$H R-p Q C T$}

HR-pQCT images of the tibia and radius were acquired on an XtremeCT in vivo scanner (Scanco Medical AG) with a $60 \mathrm{kVp}$ source potential, $900 \mu \mathrm{A}$ tube current, and $100 \mathrm{~ms}$ integration time. An image with 110 slices, beginning $22.5 \mathrm{~mm}$ and $9.5 \mathrm{~mm}$ 
proximal to the endplate for the tibia and radius, respectively, and with an isotropic nominal resolution of $82 \mu \mathrm{m}$ resulted from the 3-min image acquisition. Immediately after image acquisition, the images were visually evaluated for motion artifacts and scans with obvious motion artifacts were repeated.

\section{$M R$ and HR-pQCT Image Alignment}

Visualization of bone marrow in cortical porosity identified in the HR-pQCT requires precise image alignment between the MR and HR-pQCT image. Image registration is the process of finding a transformation that aligns the anatomical regions of two images. Because bone is a rigid structure, a global rigid transform applied to the MR image aligning it to HR-pQCT image is desired. Mutual information, a measure determined from the joint probability distribution of intensities, implemented as a similarity measure in a registration algorithm has been shown to efficiently identify accurate transformations between different imaging modalities. ${ }^{38,60}$ For each of the image pairs, Rview, ${ }^{52,55}$ a robust registration algorithm based on normalized mutual information, was used to determine a rigid transformation consisting of three Euler angles and a translation vector. This rigid transformation was then applied to the MR image, using bspline approximation for interpolation, to align and resample the MR image to the HR-pQCT image. Transformation and interpolation were not performed on the HR-pQCT image to ensure accurate measurement of cortical bone pore sizes. The registration and transformation were performed on a Sun workstation (Sun Ultra 40, AMD Opteron Dual Core processor,
2.4 GHz, 8 GB RAM, Sun Microsystems, CA). The computation time for the registration was approximately $1 \mathrm{~min}$ and for the application of the transformation was approximately $5 \mathrm{~min}$.

The robustness of the registration was assessed using a technique similar to that presented by Studholme et $a .^{54}$ using one of the tibia and one of the radius image sets. We defined a series of 30 misalignments corresponding to a translation of $5 \mathrm{~mm}$ and a rotation of $5^{\circ}$ to simulate different starting values. Each of the 30 misalignments was determined by randomly selecting a point on the surface of spheres in translational and rotational parameter space. Each misalignment was selected as the initial starting guess for the registration. The resulting 30 transforms were recorded and applied to each pixel to calculate the root mean square error for each pixel in the volume.

\section{Cortical Porosity Analysis}

The HR-pQCT data were segmented, and the periosteal surface was identified using evaluation routines provided by the manufacturer. A semi-automated edge-defining algorithm was applied to the original grayscale image to identify the periosteal surface. Then, a previously described postprocessing proto$\mathrm{col}^{19,33}$ was performed in which a low-pass Gaussian filter $(\sigma=2.0$, support $=3)$ and then a fixed global threshold was applied to identify the cortical compartment and segment the bone from background.

Code developed using MATLAB identified the cortical porosity in the HR-pQCT image and determined the existence of bone marrow in the aligned $\mathrm{MR}$ image. The process is depicted in Fig. 1. To locate

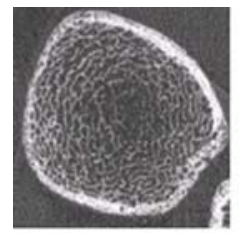

HR-PQCT

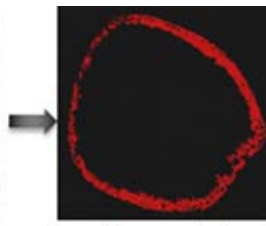

Segmented Cortical Region

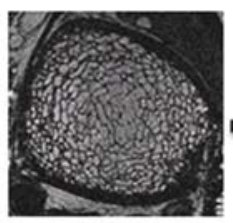

Registered and Resampled MRI

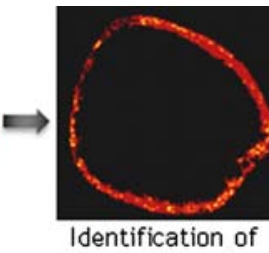

Identification of Cortical Porosity

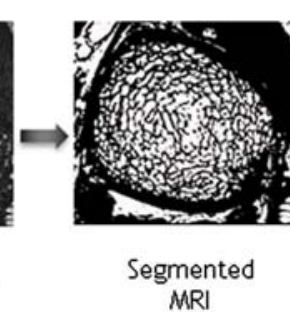

- Cortical Bone Cortical Porosity

- Cortical Porosity + Marrow

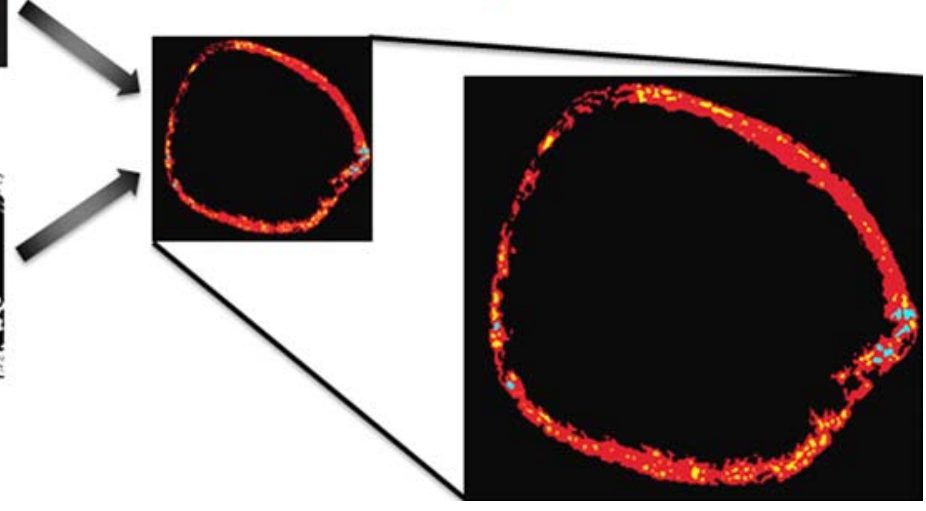

FIGURE 1. Diagram demonstrating image processing steps. The HR-pQCT image is smoothed and thresholded to identify the cortical compartment. The bone marrow in the MR image is segmented using histogram thresholding. The segmented MR image and the cortical region of the segmented HR-pQCT image are then combined. 
cortical porosity, a two-dimensional mathematical morphologic hole filling algorithm ${ }^{59}$ identified groups of connected components within the cortex. The area of each connected component (each cortical pore) was determined and the percent of cortical porosity for each slice was calculated by dividing the total area of the connected components by the entire cortical area (area of the cortical bone plus area of cortical porosity). The total number of cortical pores, the area of each cortical pore, and the mean cortical pore area for each subject were recorded. MR images were segmented to identify bone marrow using a histogram thresholding technique ${ }^{44}$ generally implemented to identify bone marrow in the trabecular region. Segmented HR-pQCT images with cortical porosity identified were then subtracted from the segmented and aligned MR images to highlight cortical porosity filled with marrow. The number of cortical pores with marrow, the size of area cortical pore with marrow, and the mean cortical pore area with marrow were recorded for each subject. The percent of cortical porosity filled with marrow was calculated by dividing the number of cortical pores with marrow by the total number of cortical pores for each subject.

\section{Statistical Analysis}

The statistical analysis was performed using JMP Version 7 software (SAS Institute, Cary, NC). The Shapiro-Wilk $W$ test was used to test the normality of the data and nonparametric methods were employed for all analyses of measurements that were not normally distributed. The significance of differences in the measurements between tibia and radius were determined using Wilcoxon signed rank test with significance set at $p<0.05$. To examine relationships between measurements, linear regression analyses were performed and Spearman's coefficient, $r$, was obtained for the correlation. The change in measurements along the length of the imaged region was determined using regression analysis. A linear-fit was used for the tibia, and a polynomial fit was used for the radius.

\section{RESULTS}

By visual assessment registration of the MR image to the HR-pQCT image was successful for all of the tibia and radius image datasets. A representative tibia and radius example are shown in Fig. 2. For the tibia, mean translations in $x, y, z$ were $0.10 \mathrm{~mm} \pm 1.61$, $-2.75 \mathrm{~mm} \pm 2.83,3.06 \mathrm{~mm} \pm 2.92$ and mean rotations in $x, y, z$ were $5.16^{\circ} \pm 3.55,-1.32^{\circ} \pm 2.57,-2.60^{\circ} \pm$ 6.49. For the radius, mean translations in $x, y, z$ were $5.00 \mathrm{~mm} \pm 4.92,-4.09 \mathrm{~mm} \pm 4.47,-2.13 \mathrm{~mm} \pm 2.91$ and mean rotations in $x, y, z$ were $-4.79^{\circ} \pm 5.35$, $174.57^{\circ} \pm 3.80,77.97^{\circ} \pm 8.93$. The large rotations in $y$ and $z$ for the radius are due to the different positioning required between HR-pQCT and MR scans. Therefore, all registrations for the radius were initialized with a starting estimate for the transformation of $180^{\circ}$ in $y$ and $80^{\circ}$ in $z$. The results from the assessment of the
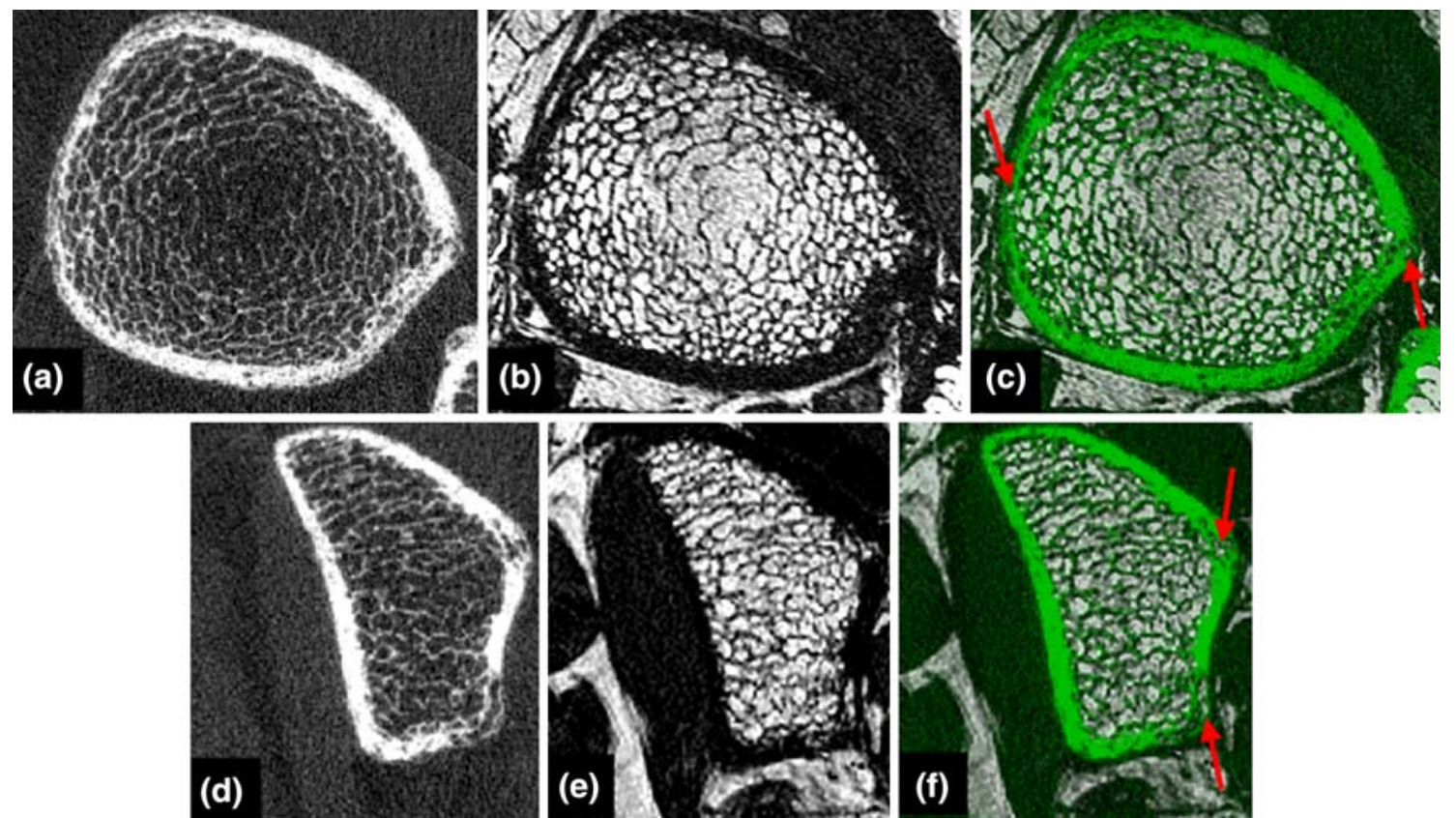

FIGURE 2. Representative tibia (a-c) and (d-f) radius images. (a, d) HR-pQCT image, (b, e) registered MR image, and (c, f) overlay between HR-pQCT (green) and MR image with cortical porosity containing bone marrow highlighted with red arrows. 
robustness of the registration showed that the RMS error for initial misalignments of $5 \mathrm{~mm}$ and $5^{\circ}$ ranged from 0.029 to $1.892 \mathrm{~mm}$ (mean $0.420 \mathrm{~mm} \pm 0.912$ ) with the larger RMS errors at the periphery of the image volume. Therefore, as long as the initial misalignment was within $5 \mathrm{~mm}$ and $5^{\circ}$, the registration was successful within approximately $0.420 \mathrm{~mm}$. However, ten of the tibia sets and 21 of the radius sets had initial misalignments beyond $5 \mathrm{~mm}$ and $5^{\circ}$ and registration was repeated with manual starting estimates to ensure an initial misalignment within the acceptable range for successful registration.

Cortical porosity was evident in all of the HR-pQCT images. The histograms for all subjects' percent cortical porosity, total number of cortical pores, and mean cortical pore area are shown in Fig. 3. A Shapiro-Wilk $W$ test for normality showed that all measurements had non-normal distributions except the total number of cortical pores for the tibia and a Wilcoxon signed rank test with significance set at $p<0.05$ showed that all measurements were significantly different between the tibia and radius. The total number of cortical pores detected within each subject for the tibia ranged from 2805 to 13,272 with a median of 7825 (interquartile range, 5909-9841) and the radius ranged from 397 to 4110 with a median of 1517 (interquartile range, 1058-1884) (Table 1). The median percent of cortical porosity for all subjects for the tibia was $3.9 \%$ (interquartile range, $3.1-4.9 \%$ ) and for the radius was $1.5 \%$ (interquartile range, $1.1-2.1 \%$ ). The mean cortical pore area for each subject is shown in Fig. 3, and the distribution of the areas of all the pores observed are shown in Fig. 4. The median area of the cortical pores for the tibia was $0.053 \mathrm{~mm}^{2}$ (interquartile range, $0.048-0.066 \mathrm{~mm}^{2}$ ) and for the radius was $0.046 \mathrm{~mm}^{2}$ (interquartile range, $0.0422-0.052 \mathrm{~mm}^{2}$ ).

Cortical porosity was observed both containing and not containing bone marrow in all subjects in both the tibia and radius. Figure 5 shows histograms for all subjects' percent cortical porosity containing marrow, the number of cortical pores containing bone marrow, and the mean area of cortical pores containing marrow. A Shapiro-Wilk $W$ test for normality showed that all measurements had non-normal distributions and a Wilcoxon signed rank test with significance set at $p<0.05$ showed that all measurements were significantly different between the tibia and radius. The number of cortical pores containing marrow for the tibia ranged from 152 to 2145 with a median of 631 (interquartile range, 412-1069) and for the radius ranged from 122 to 699 with a median of 258 (interquartile range, 177-328) (Table 1). The median percent cortical porosity containing marrow for all subjects for the tibia was $8.5 \%$ (interquartile range, $5.3-13.5 \%$ ) and for the radius was $17.0 \%$ (interquartile range, $12.9-24.8 \%$ ). The distribution of the areas of all cortical pores

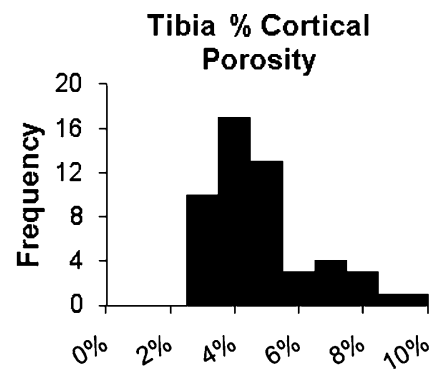

Percent

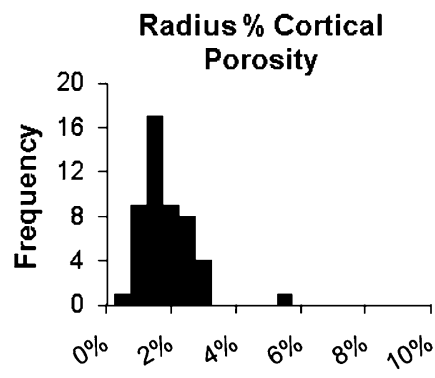

Percent

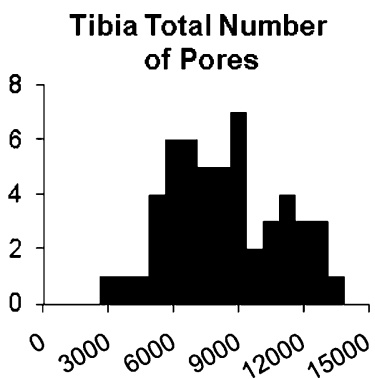

Number of Cortical Pores

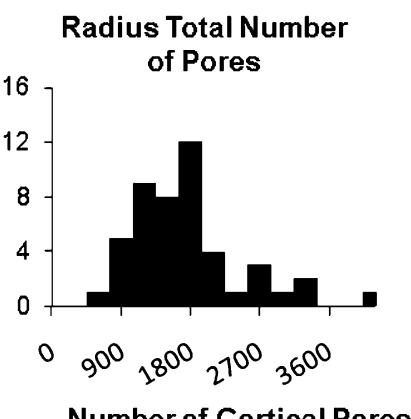

Number of Cortical Pores

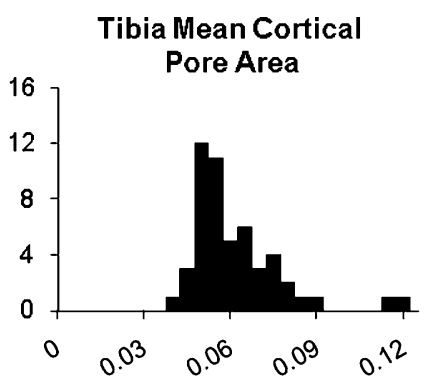

Average Area $\left(\mathrm{mm}^{2}\right)$

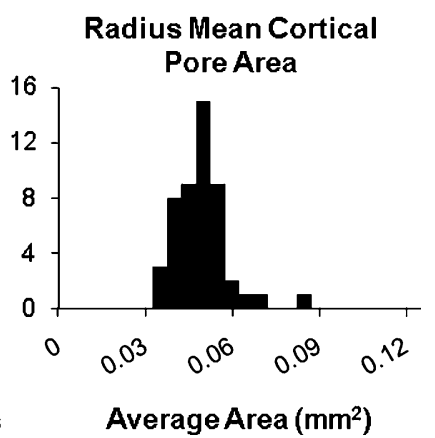

FIGURE 3. Histograms for percent cortical porosity, total number of cortical pores, and mean cortical pore area for all tibiae (top row) and radii (bottom row). All measurements are significantly different between the tibia and the radius $(p<0.001)$. 
TABLE 1. Minimum (Min), maximum (Max), median, interquartile range, mean, and standard deviation (SD) values for cortical porosity, number of cortical pores, and mean cortical pore area.

\begin{tabular}{|c|c|c|c|c|c|c|c|c|c|c|c|c|}
\hline & \multicolumn{6}{|c|}{ Tibia } & \multicolumn{6}{|c|}{ Radius } \\
\hline & Min & Max & Median & $\begin{array}{l}\text { Interquartile } \\
\text { range }\end{array}$ & Mean & SD & Min & Max & Median & $\begin{array}{l}\text { Interquartile } \\
\text { range }\end{array}$ & Mean & SD \\
\hline Cortical porosity (\%) & 2.1 & 9.2 & 3.9 & $3.1-4.9$ & 4.4 & 1.6 & 0.4 & 5.0 & 1.5 & $1.1-2.1$ & 1.6 & 0.8 \\
\hline Number of pores & 2805 & 13272 & 7825 & 5909-9841 & 8027 & 2567 & 397 & 4110 & 1517 & $1058-1884$ & 1615 & 783 \\
\hline Mean cortical pore area $\left(\mathrm{mm}^{2}\right)$ & 0.039 & 0.119 & 0.053 & $0.048-0.066$ & 0.059 & 0.016 & 0.032 & 0.08 & 0.046 & $0.042-0.052$ & 0.046 & 0.008 \\
\hline Cortical porosity with marrow (\%) & 1.6 & 27.0 & 8.5 & $5.3-13.5$ & 10.0 & 6.3 & 4.9 & 41.8 & 17.0 & $12.9-24.8$ & 19.1 & 8.9 \\
\hline Number of pores with marrow & 152 & 2145 & 631 & 412-1069 & 765 & 494 & 122 & 699 & 258 & 177-328 & 266 & 108 \\
\hline $\begin{array}{l}\text { Mean cortical pore area with } \\
\text { marrow }\left(\mathrm{mm}^{2}\right)\end{array}$ & 0.057 & 0.314 & 0.128 & $0.108-0.176$ & 0.144 & 0.053 & 0.027 & 0.238 & 0.075 & $0.059-0.086$ & 0.075 & 0.03 \\
\hline
\end{tabular}

A Shapiro-Wilk $W$ test for normality showed that only the number of cortical pores for the tibia had a normal distribution.
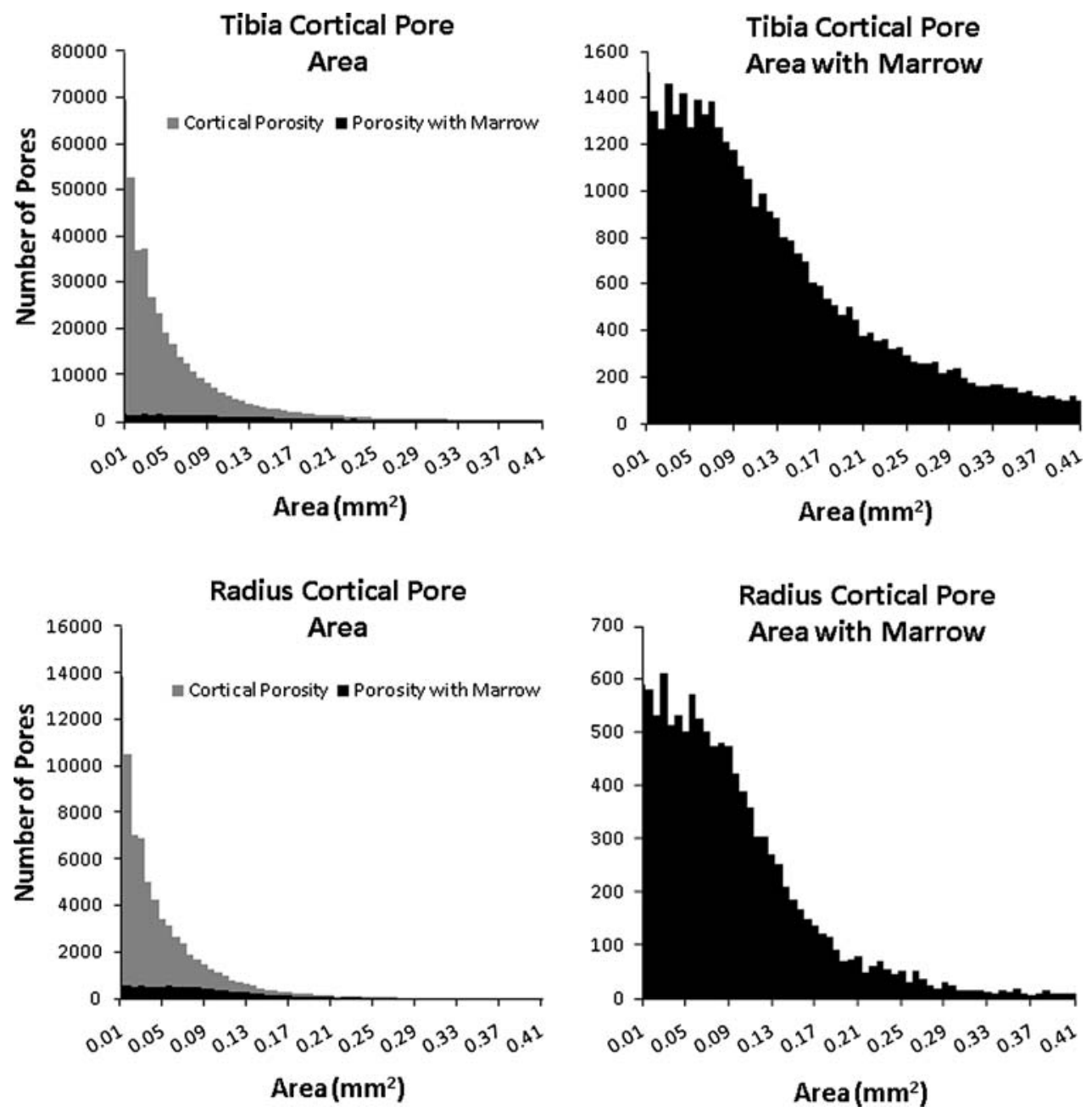

FIGURE 4. Histograms of the area for all of the cortical pores identified and all cortical pores with marrow for all tibiae (top row) and radii (bottom row).

containing marrow observed is shown in Fig. 4, and the mean cortical pore area for each subject is shown in Fig. 5. The median area of cortical pores containing marrow for the tibia was $0.128 \mathrm{~mm}^{2}$ (interquartile range, $0.108-0.176 \mathrm{~mm}^{2}$ ) and for the radius $0.075 \mathrm{~mm}^{2}$ (interquartile range, $0.059-0.086 \mathrm{~mm}^{2}$ ).

The mean area of cortical pores containing marrow was significantly higher than the mean area of all the 

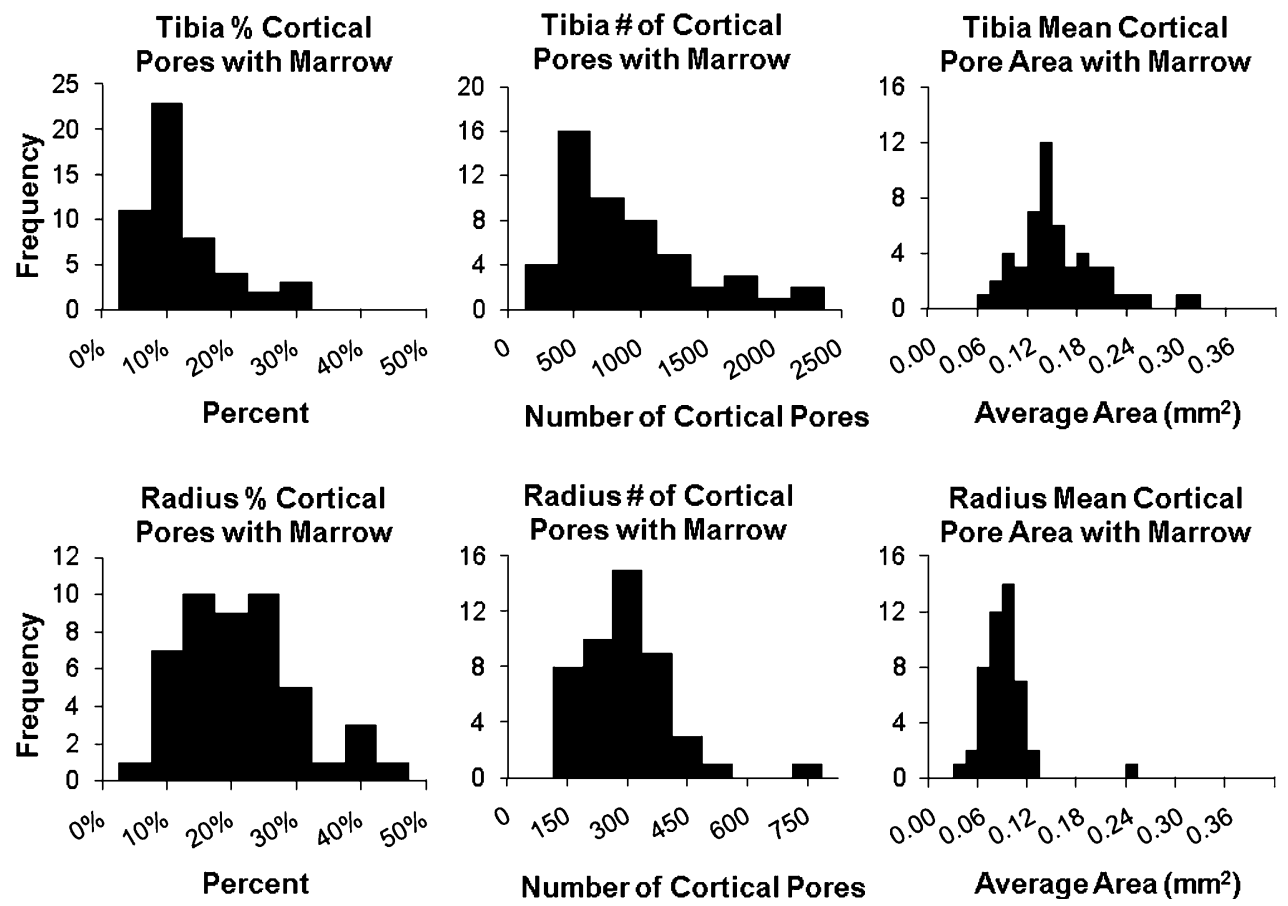

FIGURE 5. Histograms for percent cortical porosity containing marrow, total number of cortical pores containing marrow, and mean cortical pore area containing marrow for all tibiae (top row) and radii (bottom row). All measurements are significantly different between the tibia and the radius $(p<0.001)$.

cortical pores $(p<0.001)$ for both the radius and tibia. The mean area of all cortical pores within a subject ranged from 0.039 to $0.119 \mathrm{~mm}^{2}$ in the tibia and from 0.032 to $0.080 \mathrm{~mm}^{2}$ in the radius while the mean area of cortical pores containing marrow ranged from $0.057 \mathrm{~mm}^{2}$ to $0.314 \mathrm{~mm}^{2}$ in the tibia and from $0.027 \mathrm{~mm}^{2}$ to $0.238 \mathrm{~mm}^{2}$ in the radius (Table 1). However, there was no relationship between the percent of cortical porosity and the percent cortical pores that contained marrow (tibia $p=0.16$; radius $p=0.09$ ) nor the mean cortical pore area and the percent of cortical pores containing marrow (tibia $p=0.18$; radius $p=0.10$ ).

In Fig. 6, the mean number of cortical pores and the mean number of cortical pores with marrow are plotted to show variations as a function of distance along the shaft (from distal to proximal). Within the $8.2 \mathrm{~mm}$ of cortical shaft analyzed, there was a linear increase in the tibia $(p<0.001)$ and a nonlinear relationship $(p<0.001)$ in the radius for both the number of cortical pores and the number of cortical pores containing marrow with distance along the shaft. Figure 7 visually demonstrates the variations in cortical porosity in one subject in which there are fewer cortical pores in the most distal slice than in the most proximal slice. Additionally there was no significant trend for cortical pore area variations in the radius or the tibia $(p>0.05)$.

\section{DISCUSSION}

In this study, we examined cortical porosity using images of both HR-pQCT and MR of the distal tibia and distal radius of postmenopausal osteopenic women. All HR-pQCT images contained cortical porosity in both the tibia and the radius. By combining the HR-pQCT and MR images, the data revealed that all subjects had cortical pores which both contained and did not contain bone marrow. The results suggest that cortical pore spaces may contain different fluids. This implies that there may be more than one mechanism for the development of cortical porosity and more than one type of bone fluid present in cortical pores.

The registration method implemented in this study performed using Rview, ${ }^{52,55}$ a robust registration algorithm based on normalized mutual information. The result of the registration method, a three-dimensional rigid transform, was used to align the MR image to the HR-pQCT image. The aligned images allowed for the visualization of bone marrow in cortical pores. When the robustness of the registration was tested, registration was successful within approximately $0.420 \mathrm{~mm}$ for misregistrations $<5 \mathrm{~mm}$ and $5^{\circ}$. This paper does not assess registration accuracy in a quantitative way, because clinical data do not provide any means of determining a gold standard registration transformation. There are several factors that may 

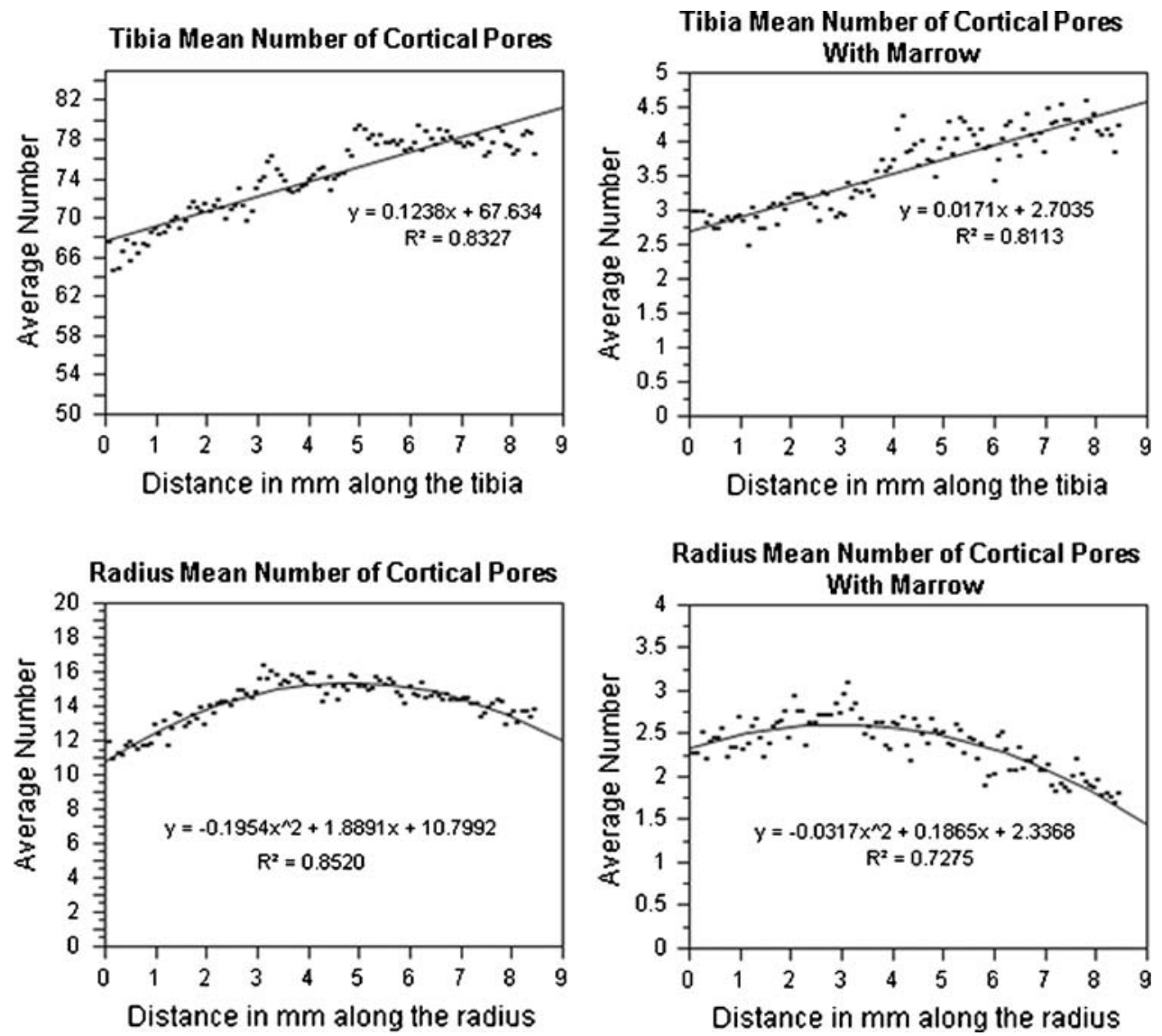

FIGURE 6. Plots of the mean number of cortical pores with and without marrow with distance along the shaft (distal to proximal) for the radius and tibia. There was a linear increasing trend determined for the tibia $(p<0.001)$ and nonlinear trend found for the radius $(p<0.001)$

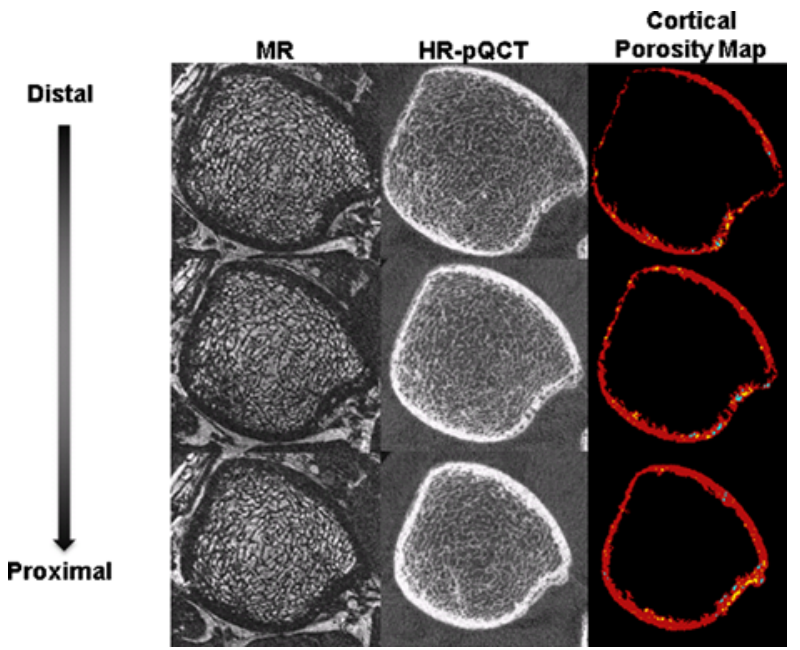

FIGURE 7. Axial images starting with the most distal slice through the most proximal slice in the analysis region for one subject. The MR images depict cortical and trabecular bone as signal void and marrow as higher in intensity, while the HR-pQCT depict bone as higher in intensity. The cortical porosity map shows cortical bone in red, cortical pores without marrow in yellow, and cortical pores with marrow in blue. impact the accuracy of the registration, including the size or amount of trabecular bone, surrounding fatty tissue, or cortical porosity. Additionally, previous studies aligning MR and CT images of heads have noted effects on registration accuracy due to geometric distortions, motion artifact, chemical shift artifact, or intensity distortions. ${ }^{23,26,53}$

While most previous studies have focused on ex vivo examinations of the cortical porosity of the femoral shaft, ${ }^{7,14,28}$ this study is the first study to examine cortical porosity of the tibia and radius using two complementary in vivo imaging techniques. Jordan et al. ${ }^{28}$ found a mean percent cortical porosity of $10.72 \% \pm 4.2$ in femoral shaft biopsies using histology sections. Similarly Cooper et al. ${ }^{14}$ reported a mean percent cortical porosity of $9.82 \% \pm 9.19$ in femoral shaft specimens from females using micro-CT imaging and morphological analysis. The percent cortical porosity in this study is lower, $4.36 \% \pm 1.6$ for the tibia and $1.6 \% \pm 0.8$ for the radius. The reason for the difference is likely due to the difference in imaging and analysis methods, anatomical region analyzed, or fracture 
status. Histology sections have a much higher resolution than HR-pQCT and can therefore visualize smaller cortical pores, which accounts for the higher porosity measurement using histology sections. Due to resolution constraints, the smallest pore area that can be measured on the HR-pQCT is $0.0067 \mathrm{~mm}^{2}$, corresponding to a diameter of $82 \mu \mathrm{m}$, while cortical porosity at the scale of lacunae and canaliculi can have diameters as small as $10 \mu \mathrm{m}$ and $0.5 \mu \mathrm{m}$, respectively. ${ }^{41,48}$ Additionally, the femur, the tibia, and the radius have different mechanical environment and may therefore have different metabolic activities which may illustrate why the radius has a lower percent of cortical porosity. Cortical porosity in this study was determined from in vivo images of postmenopausal women who had not sustained a fracture while previous studies determined cortical porosity from fractured femoral neck biopsies of women or femoral midshaft specimens of male cadavers. This difference in fracture status or sex may impact the percent of cortical porosity observed.

Alendronate and risedronate are second- and thirdgeneration bisphosphonates that have been shown to reduce hip fractures in postmenopausal women. ${ }^{8,17,25}$ Studies have demonstrated that alendronate inhibits osteoclastic bone resorption, reduces bone turnover, increases hip BMD, produces more uniform mineralization in cortical bone, and decreases cortical porosity. ${ }^{8,17,34,50}$ Both Roschger et al. ${ }^{50}$ and Borah et al. ${ }^{9}$ observed a reduction in cortical porosity in an antiresorptive therapy group compared with a placebotreated group which highlights the importance of cortical porosity in overall bone quality maintenance. Determining how cortical porosity changes in response to alendronate treatment is important in assessing drug efficacy. However, the images in this study were acquired prior to alendronate treatment and longitudinal changes in the amount of cortical porosity and the visualization of bone marrow within cortical pores will be assessed after follow-up analysis is performed.

Cortical porosity was visualized with and without bone marrow in every subject in this study. There was a large range in the percent of cortical porosity with bone marrow across subjects. While the amount of cortical porosity ranged $2.1-9.2 \%$ in the tibia and $0.4-5.0 \%$ in the radius across all subjects, the range of percent of porosity containing marrow was much greater, $1.6-21.0 \%$ in the tibia and $4.9-41.8 \%$ in the radius. This suggests that while the amount of cortical porosity did not vary greatly between subjects, the type of cortical pore, containing bone marrow vs. not containing bone marrow, varied highly between subjects. Additionally, the number of cortical pores containing bone marrow did not depend on the percent of cortical porosity and pores for all visible cortical pore areas $\left(>0.0067 \mathrm{~mm}^{2}\right)$ were observed with and without bone marrow (Fig. 4). The regional variation in cortical porosity observed in this study (Fig. 6) may have considerable impact when assessing cortical porosity using a single-slice technique and also demonstrates the importance of consistent analysis regions in longitudinal or cross-sectional studies investigating cortical porosity.

Bone fluids within the bone cavities of the cortical bone have been assumed to be homogeneous across all cavities. ${ }^{16}$ Although the viscosity and compressibility of the bone fluid has never been measured, it is assumed to behave like salt water. ${ }^{16,35,43}$ However, this study suggests there may exist variability in bone fluid composition. Since the viscosity and compressibility of the bone fluid impact the hydrostatic strengthening (HS) of cortical bone, ${ }^{35,36}$ knowledge of bone fluid composition and distribution may help improve the accuracy of HS models and further help predicts bone strength.

Bone marrow consists of both hematopoietic (red) and fatty (yellow) components. Red marrow consists of approximately $40 \%$ water, $40 \%$ fat, and $20 \%$ protein while yellow marrow consists of approximately $15 \%$ water, $80 \%$ fat, and $5 \%$ protein. ${ }^{57}$ Normal physiological conversion to the adult pattern, conversion from red to yellow marrow, completes by the age of 25 at which time red marrow is predominantly concentrated in the axial skeleton and yellow marrow is predominately concentrated in peripheral regions such as the tibia and radius. ${ }^{42,46}$ When imaging bone marrow using $\mathrm{MR}$, fat is the predominate contributor due to its short $\mathrm{T} 1$ relaxation time compared with the longer $\mathrm{T} 1$ relaxation time of water. ${ }^{61}$ Due to this difference, yellow marrow appears much brighter on an MR image with $\mathrm{T} 1$ weighting. ${ }^{58}$ The assumption for peripheral regions, such as the tibia and radius, is that all marrow present is yellow marrow. However, the possibility that red marrow may be present cannot be eliminated and exact percentage of fat within the marrow is unknown, as well as the amount of saturated, monounsaturated, and polyunsaturated triglycerides. Proton MR spectroscopy is a promising tool for determining the composition of bone marrow ${ }^{37,64}$ and Ultrashort TE (UTE) pulse sequences have been shown to visualize tissues with a very short $\mathrm{T} 2$ relaxation component ${ }^{24,49}$ which may be helpful in future studies to determine the exact constituents of the bone marrow fluid in cortical pores. In this study, cortical pores visualized without bone marrow may be likely to contain bone fluid with a high water content and therefore a longer $\mathrm{T} 1$ which results in a loss of MR signal.

Cortical pores containing bone marrow are not likely to be large haverian canals with blood vessels but rather resorption spaces that been infiltrated by bone marrow. Most likely these are resorption spaces in the cortex that have broken the endosteal boundary 
allowing the infiltration of bone marrow. While this study evaluated cortical porosity using two-dimensional techniques, a three-dimensional analysis of the cortical porosity may clarify whether cortical pores containing marrow connect to the trabecular bone marrow compartment. Since cortical pores may cross multiple slices, it is expected that a three-dimensional analysis of the cortical porosity may results in a smaller number of cortical pores. Additionally, a previous study has shown that cortical bone loss may vary spatially. ${ }^{6}$ A future direction may be to assess the primary location of cortical porosity and the spatial distribution of cortical pores containing marrow vs. cortical pores not containing bone marrow. Previous studies have demonstrated that age is highly correlated with increased cortical porosity, ${ }^{11,14,20,56}$ therefore an additional future direction may be to assess the relationship between age and the percent of cortical pores containing marrow.

There were several limitations in this study related to imaging and postprocessing restrictions. Scanning a region containing a high trabecular density causes the cortical bone segmentation to be more difficult than if the midshaft had been used. However, the regions scanned in this study were chosen to allow for cortical and trabecular bone analysis. Davis et al. ${ }^{19}$ describe inaccuracies of the cortical bone segmentation which is highly dependent on the Gaussian blurring step to smooth the finer trabecular bone structure. This dilution may cause thin segments of the cortex and highly porous regions of cortical bone to be identified as trabecular bone. Therefore, cortical pores in this study that were located inside thin segments of the cortex, within highly porous regions, or closer to the endosteal surface may have been excluded and this study may underestimate the actual amount of cortical porosity. Additionally, MR images are acquired at a lower resolution than the HR-pQCT images and then resampled to match the HR-pQCT image resolution. Therefore, due to partial volume effects, marrow in some pores may not have been visualized while marrow in cortical pore sizes $<0.024 \mathrm{~mm}^{2}$ (the resolution of the original MR image) may be exaggerated. However, we propose that our technique is able to successfully visualize the presence of bone marrow in the majority of cortical pores and within these cortical pores, estimate the number and size of cortical pores containing bone marrow.

This study observes cortical porosity in vivo in postmenopausal osteopenic women and combines data from two different imaging modalities, HR-pQCT and MRI to further study the nature of cortical porosity. Data suggest that cortical porosity in the distal tibia and distal radius are prevalent in this population and that the constituents of cortical pore fluid may vary. The number of cortical pores containing bone marrow varies between subjects but is not dependent on the amount of cortical porosity. Future investigation will be required to fully examine and interpret the observations in this study and to determine whether the spatial distribution of bone marrow in cortical porosity has an impact on bone strength.

\section{ACKNOWLEDGMENTS}

The authors thank David Newitt, Benedict Hyun, Andrew Burghardt, Thelma Munoz, Jingyi Yu, Nicole Cheng, Melissa Guan, and Ayako Suzuki for contributions in recruiting subjects, image acquisition, and database management. We thank Andres Laib and Scanco Medical AG for providing consultation and software development support. We also thank Anne de Papp and Merck \& Co for their support. This work is funded by a grant from Merck \& Co. and by ARCS and Evnin-Wright Fellowships.

\section{CONFLICT OF INTEREST}

The authors have no conflicts of interest; however, the study was partially funded by Merck \& Co.

\section{OPEN ACCESS}

This article is distributed under the terms of the Creative Commons Attribution Noncommercial License which permits any noncommercial use, distribution, and reproduction in any medium, provided the original author(s) and source are credited.

\section{REFERENCES}

\footnotetext{
${ }^{1}$ Aguado, F., M. Revilla, L. F. Villa, and H. Rico. Cortical bone resorption in osteoporosis. Calcif. Tissue Int. 60(4):323-326, 1997.

${ }^{2}$ Augat, P., H. Reeb, and L. E. Claes. Prediction of fracture load at different skeletal sites by geometric properties of the cortical shell. J. Bone Miner. Res. 11(9):1356-1363, 1996.

${ }^{3}$ Banerjee, S., E. T. Han, R. Krug, D. C. Newitt, and S. Majumdar. Application of refocused steady-state freeprecession methods at 1.5 and $3 \mathrm{~T}$ to in vivo high-resolution MRI of trabecular bone: simulations and experiments. J. Magn. Reson. Imaging 21(6):818-825, 2005.

${ }^{4}$ Bell, K. L., N. Loveridge, G. R. Jordan, J. Power, C. R. Constant, and J. Reeve. A novel mechanism for induction of increased cortical porosity in cases of intracapsular hip fracture. Bone 27(2):297-304, 2000.

${ }^{5}$ Bell, K. L., N. Loveridge, J. Power, N. Garrahan, B. F. Meggitt, and J. Reeve. Regional differences in cortical
} 
porosity in the fractured femoral neck. Bone 24(1):57-64, 1997.

${ }^{6}$ Bell, K. L., N. Loveridge, J. Power, N. Garrahan, M. Stanton, M. Lunt, B. F. Meggitt, and J. Reeve. Structure of the femoral neck in hip fracture: cortical bone loss in the inferoanterior to superoposterior axis. J. Bone Miner. Res. 14(1):111-119, 1999.

${ }^{7}$ Bell, K. L., N. Loveridge, J. Reeve, C. D. Thomas, S. A. Feik, and J. G. Clement. Super-osteons (remodeling clusters) in the cortex of the femoral shaft: influence of age and gender. Anat. Rec. 264(4):378-386, 2001.

${ }^{8}$ Black, D. M., S. R. Cummings, D. B. Karpf, J. A. Cauley, D. E. Thompson, M. C. Nevitt, D. C. Bauer, H. K. Genant, W. L. Haskell, R. Marcus, S. M. Ott, J. C. Torner, S. A. Quandt, T. F. Reiss, and K. E. Ensrud. Randomised trial of effect of alendronate on risk of fracture in women with existing vertebral fractures. Fracture Intervention Trial Research Group. Lancet 348(9041):1535-1541, 1996.

${ }^{9}$ Borah, B., T. Dufresne, J. Nurre, P. Chmielewski, R. Phipps, L. Wagner, M. Bouxsein, R. Zebaze, and E. Seeman. Risedronate Reduces Intracortical Porosity in Women with Osteoporosis. Vienna, Austria: European Calcified Tissue Society, 2009.

${ }^{10}$ Borah, B., T. Dufresne, J. Nurre, R. Phipps, P. Chmielewski, L. Wagner, M. Lundy, M. Bouxsein, R. Zebaze, and E. Seeman. Risedronate reduces intracortical porosity in women with osteoporosis. J. Bone Miner. Res. 2009, in press.

${ }^{11}$ Bousson, V., A. Meunier, C. Bergot, E. Vicaut, M. A. Rocha, M. H. Morais, A. M. Laval-Jeantet, and J. D. Laredo. Distribution of intracortical porosity in human midfemoral cortex by age and gender. J. Bone Miner. Res. 16(7):1308-1317, 2001.

${ }^{12}$ Buie, H. R., G. M. Campbell, R. J. Klinck, J. A. MacNeil, and S. K. Boyd. Automatic segmentation of cortical and trabecular compartments based on a dual threshold technique for in vivo micro-CT bone analysis. Bone 41(4):505515, 2007.

${ }^{13}$ Burstein, A. H., D. T. Reilly, and M. Martens. Aging of bone tissue: mechanical properties. J. Bone Jt. Surg. Am. 58(1):82-86, 1976.

${ }^{14}$ Cooper, D. M., C. D. Thomas, J. G. Clement, A. L. Turinsky, C. W. Sensen, and B. Hallgrimsson. Agedependent change in the $3 \mathrm{D}$ structure of cortical porosity at the human femoral midshaft. Bone 40(4):957-965, 2007.

${ }^{15}$ Cooper, D. M., A. L. Turinsky, C. W. Sensen, and B. Hallgrimsson. Quantitative 3D analysis of the canal network in cortical bone by micro-computed tomography. Anat. Rec. B New Anat. 274(1):169-179, 2003.

${ }^{16}$ Cowin, S. C. Bone poroelasticity. J. Biomech. 32(3):217238, 1999.

${ }^{17}$ Cummings, S. R., D. M. Black, D. E. Thompson, W. B. Applegate, E. Barrett-Connor, T. A. Musliner, L. Palermo, R. Prineas, S. M. Rubin, J. C. Scott, T. Vogt, R. Wallace, A. J. Yates, and A. Z. LaCroix. Effect of alendronate on risk of fracture in women with low bone density but without vertebral fractures: results from the Fracture Intervention Trial. JAMA 280(24):2077-2082, 1998.

${ }^{18}$ Currey, J. D. The effect of porosity and mineral content on the Young's modulus of elasticity of compact bone. J. Biomech. 21(2):131-139, 1988.

${ }^{19}$ Davis, K. A., A. J. Burghardt, T. M. Link, and S. Majumdar. The effects of geometric and threshold definitions on cortical bone metrics assessed by in vivo highresolution peripheral quantitative computed tomography. Calcif. Tissue Int. 81(5):364-371, 2007.
${ }^{20}$ Feik, S. A., C. D. Thomas, and J. G. Clement. Age-related changes in cortical porosity of the midshaft of the human femur. J. Anat. 191(Pt 3):407-416, 1997.

${ }^{21}$ Genant, H. K., C. C. Gluer, and J. C. Lotz. Gender differences in bone density, skeletal geometry, and fracture biomechanics. Radiology 190(3):636-640, 1994.

${ }^{22}$ Greenspan, S. L., T. J. Beck, N. M. Resnick, R. Bhattacharya, and R. A. Parker. Effect of hormone replacement, alendronate, or combination therapy on hip structural geometry: a 3year, double-blind, placebo-controlled clinical trial. J. Bone Miner. Res. 20(9):1525-1532, 2005.

${ }^{23}$ Holden, M., D. L. Hill, E. R. Denton, J. M. Jarosz, T. C. Cox, T. Rohlfing, J. Goodey, and D. J. Hawkes. Voxel similarity measures for 3-D serial MR brain image registration. IEEE Trans. Med. Imaging 19(2):94-102, 2000.

${ }^{24}$ Holmes, J. E., and G. M. Bydder. MR imaging with ultrashort TE (UTE) pulse sequences: basic principles. Radiography 11:163-174, 2005.

${ }^{25}$ Iwamoto, J., Y. Sato, T. Takeda, and H. Matsumoto. Hip fracture protection by alendronate treatment in postmenopausal women with osteoporosis: a review of the literature. Clin. Interv. Aging 3(3):483-489, 2008.

${ }^{26}$ Jenkinson, M., P. Bannister, M. Brady, and S. Smith. Improved optimization for the robust and accurate linear registration and motion correction of brain images. $\mathrm{Neu}$ roimage 17(2):825-841, 2002.

${ }^{27}$ Jepsen, K. J., The aging cortex: to crack or not to crack. Osteoporos. Int. 14(Suppl 5):S57-S62; discussion S63-66, 2003.

${ }^{28}$ Jordan, G. R., N. Loveridge, K. L. Bell, J. Power, N. Rushton, and J. Reeve. Spatial clustering of remodeling osteons in the femoral neck cortex: a cause of weakness in hip fracture? Bone 26(3):305-313, 2000.

${ }^{29}$ Kasra, M., and M. D. Grynpas. Static and dynamic finite element analyses of an idealized structural model of vertebral trabecular bone. J. Biomech. Eng. 120(2):267-272, 1998.

${ }^{30}$ Kasra, M., and M. D. Grynpas. On shear properties of trabecular bone under torsional loading: effects of bone marrow and strain rate. J. Biomech. 40(13):2898-2903, 2007.

${ }^{31}$ Kazakia, G. J., B. Hyun, A. J. Burghardt, R. Krug, D. C. Newitt, A. E. de Papp, T. M. Link, and S. Majumdar. In vivo determination of bone structure in postmenopausal women: a comparison of HR-pQCT and high-field MR imaging. J. Bone Miner. Res. 23(4):463-474, 2008.

${ }^{32}$ Krug, R., E. T. Han, S. Banerjee, and S. Majumdar. Fully balanced steady-state 3D-spin-echo (bSSSE) imaging at 3 Tesla. Magn. Reson. Med. 56(5):1033-1040, 2006.

${ }^{33}$ Laib, A., H. J. Hauselmann, and P. Ruegsegger. In vivo high resolution 3D-QCT of the human forearm. Technol. Health Care 6(5-6):329-337, 1998.

${ }^{34}$ Liberman, U. A., S. R. Weiss, J. Broll, H. W. Minne, H. Quan, N. H. Bell, J. Rodriguez-Portales, R. W. Downs, Jr., J. Dequeker, and M. Favus. Effect of oral alendronate on bone mineral density and the incidence of fractures in postmenopausal osteoporosis. The Alendronate Phase III Osteoporosis Treatment Study Group. N. Engl. J. Med. 333(22):1437-1443, 1995.

${ }^{35}$ Liebschner, M. A., and T. S. Keller. Hydraulic strengthening affects the stiffness and strength of cortical bone. Ann. Biomed. Eng. 33(1):26-38, 2005.

${ }^{36}$ Liebschner, M. A., and T. S. Keller. The importance of permeability constant in hydraulic strengthening of cortical bone. In: Proceedings of the 4th ASME Summer Bioengineering Conference, Big Sky, Montana, p. 321, 1999. 
${ }^{37}$ Liney, G. P., C. P. Bernard, D. J. Manton, L. W. Turnbull, and C. M. Langton. Age, gender, and skeletal variation in bone marrow composition: a preliminary study at 3.0 Tesla. J. Magn. Reson. Imaging 26(3):787-793, 2007.

${ }^{38}$ Maes, F., A. Collignon, D. Vandermeulen, G. Marchal, and P. Suetens. Multimodality image registration by maximization of mutual information. IEEE Trans. Med. Imaging 16(2):187-198, 1997.

${ }^{39}$ Martin, R. B., J. C. Pickett, and S. Zinaich. Studies of skeletal remodeling in aging men. Clin. Orthop. Relat. Res. 149:268-282, 1980.

${ }^{40}$ McCalden, R. W., J. A. McGeough, M. B. Barker, and C. M. Court-Brown. Age-related changes in the tensile properties of cortical bone. The relative importance of changes in porosity, mineralization, and microstructure. J. Bone Jt. Surg. Am. 75(8):1193-1205, 1993.

${ }^{41}$ McCreadie, B. R., S. J. Hollister, M. B. Schaffler, and S. A. Goldstein. Osteocyte lacuna size and shape in women with and without osteoporotic fracture. J. Biomech. 37(4):563572, 2004.

${ }^{42}$ Mitchell, D. G., V. M. Rao, M. Dalinka, C. E. Spritzer, L. Axel, W. Gefter, M. Kricun, M. E. Steinberg, and H. Y. Kressel. Hematopoietic and fatty bone marrow distribution in the normal and ischemic hip: new observations with 1.5T MR imaging. Radiology 161(1):199-202, 1986.

${ }^{43}$ Neuman, W. F., and M. W. Neuman. The Chemical Dynamics of Bone. Chicago: University of Chicago Press, 1958.

${ }^{44}$ Newitt, D. C., B. Van Rietbergen, and S. Majumdar. Processing and analysis of in vivo high-resolution MR images of trabecular bone for longitudinal studies: reproducibility of structural measures and micro-finite element analysis derived mechanical properties. Osteoporos. Int. 13:278-287, 2002.

${ }^{45}$ Ochoa, J. A., D. A. Heck, K. D. Brandt, and B. M. Hillberry. The effect of intertrabecular fluid on femoral head mechanics. J. Rheumatol. 18(4):580-584, 1991.

${ }^{46}$ Piney, A. The anatomy of the bone marrow. BMJ 2:792795, 1922.

${ }^{47}$ Power, J., N. Loveridge, A. Lyon, N. Rushton, M. Parker, and $\mathbf{J}$. Reeve. Osteoclastic cortical erosion as a determinant of subperiosteal osteoblastic bone formation in the femoral neck's response to BMU imbalance. Effects of stancerelated loading and hip fracture. Osteoporos. Int. 16(9): 1049-1056, 2005.

${ }^{48}$ Reilly, G. C., H. F. Knapp, A. Stemmer, P. Niederer, and M. L. Knothe Tate. Investigation of the morphology of the lacunocanalicular system of cortical bone using atomic force microscopy. Ann. Biomed. Eng. 29(12):1074-1081, 2001.

${ }^{49}$ Robson, M. D., P. D. Gatehouse, M. Bydder, and G. M. Bydder. Magnetic resonance: an introduction to ultrashort
TE (UTE) imaging. J. Comput. Assist. Tomogr. 27(6):825846, 2003.

${ }^{50}$ Roschger, P., S. Rinnerthaler, J. Yates, G. A. Rodan, P. Fratzl, and K. Klaushofer. Alendronate increases degree and uniformity of mineralization in cancellous bone and decreases the porosity in cortical bone of osteoporotic women. Bone 29(2):185-191, 2001.

${ }^{51}$ Schaffler, M. B., and D. B. Burr. Stiffness of compact bone: effects of porosity and density. J. Biomech. 21(1):13-16, 1988.

${ }^{52}$ Studholme, C. Available from: http://rview.colin-studholme. net, 2009.

${ }^{53}$ Studholme, C., D. L. Hill, and D. J. Hawkes. Automated 3-D registration of MR and CT images of the head. Med. Image Anal. 1(2):163-175, 1996.

${ }^{54}$ Studholme, C., D. L. Hill, and D. J. Hawkes. Automated three-dimensional registration of magnetic resonance and positron emission tomography brain images by multiresolution optimization of voxel similarity measures. Med. Phys. 24(1):25-35, 1997.

${ }^{55}$ Studholme, C., D. L. G. Hill, and D. J. Hawkes. An overlap invariant entropy measure of $3 \mathrm{D}$ medical image alignment. Pattern Recogn. 32(1):71-86, 1999.

${ }^{56}$ Thomas, C. D., S. A. Feik, and J. G. Clement. Regional variation of intracortical porosity in the midshaft of the human femur: age and sex differences. J. Anat. 206(2):115125,2005

${ }^{57}$ Trabowitz, S., and S. Davis. The bone marrow matrix. In: The Human Bone Marrow: Anatomy, Physiology, and Pathophysiology, edited by S. Trabowitz and S. Davis. Boca Raton, FL: CRC, 1982, pp. 43-76.

${ }^{58}$ Vande Berg, B. C., J. Malghem, F. E. Lecouvet, and B. Maldague. Magnetic resonance imaging of the normal bone marrow. Skeletal Radiol. 27(9):471-483, 1998.

${ }^{59}$ Vincent, L. Morphological grayscale reconstruction in image analysis: applications and efficient algorithms. IEEE Trans. Image Process. 2(2):176-201, 1993.

${ }^{60}$ Viola, P., and W. M. Wells. Alignment by maximization of mutual information. Int. J. Comp. Vis. 24(2):137-154, 1997.

${ }^{61}$ Vogler, J. B., III, and W. A. Murphy. Bone marrow imaging. Radiology 168(3):679-693, 1988.

${ }^{62}$ World Health Organization. Assessment of fracture risk and its application to screening for postmenopausal osteoporosis. Report of a WHO Study Group. WHO Tech Rep Ser., 1994.

${ }^{63}$ Yeni, Y. N., C. U. Brown, Z. Wang, and T. L. Norman. The influence of bone morphology on fracture toughness of the human femur and tibia. Bone 21(5):453-459, 1997.

${ }^{64}$ Yeung, D. K., J. F. Griffith, G. E. Antonio, F. K. Lee, J. Woo, and P. C. Leung. Osteoporosis is associated with increased marrow fat content and decreased marrow fat unsaturation: a proton MR spectroscopy study. J. Magn. Reson. Imaging 22(2):279-285, 2005. 